Research Article

\title{
Analyzing the Formation Mechanism of Cross-City Transportation Network Resilience
}

\author{
Liang Wang $\mathbb{D},{ }^{1}$ Xiaolong Xue $\mathbb{D},{ }^{2}$ Weirui Xue, ${ }^{2}$ and Beile Zhao ${ }^{1}$ \\ ${ }^{1}$ School of Maritime Economics and Management, Dalian Maritime University, 1 Linghai Road, Dalian 116026, China \\ ${ }^{2}$ School of Management, Guangzhou University, 230 Outer Ring Road, Guangzhou 510006, China \\ Correspondence should be addressed to Xiaolong Xue; xlxue@hit.edu.cn
}

Received 29 January 2021; Revised 16 February 2021; Accepted 4 March 2021; Published 12 March 2021

Academic Editor: Guangdong Wu

Copyright (c) 2021 Liang Wang et al. This is an open access article distributed under the Creative Commons Attribution License, which permits unrestricted use, distribution, and reproduction in any medium, provided the original work is properly cited.

The formation mechanism of cross-city transportation network resilience occupies an important position in cross-city transportation network resilience management. This study analyzes the constituent elements of the cross-city transportation network and their interrelationships, and the connotation of cross-city transportation network resilience is defined from the general meaning of system resilience. Combining with the connotation of cross-city transportation network resilience, the specific formation process of cross-city transportation network resilience is analyzed and summarized from three stages, including resisting disturbance, absorbing disturbance, and function recovery. Taking cross-city transportation network nodes and systems as specific objects, the static and dynamic formation path of cross-city transportation network resilience is condensed. Based on the standard linear solid model, a theoretical model is constructed and solved for revealing the formation mechanism of cross-city transportation network resilience. Finally, the theoretical model of cross-city transportation network resilience proposed in this study is used for analyzing the China railway network resilience.

\section{Introduction}

With the continuous advancement of urbanization, cities and transportation infrastructure between cities form a huge cross-city transportation network in the form of nodes and links $[1,2]$. Local disturbances faced by a single city will be amplified through cross-city transportation networks and become common disturbances in the region or even the entire cross-city transportation network [3, 4]. As an important part of the socio-technical system, cross-city transportation network should show resilience in the face of disturbances, which means cross-city transportation network can resist external disturbances and maintain certain network performance, and quickly recover to the original equilibrium state after disturbances disappearing or adjust itself to a new state according to the external environment $[5,6]$.

With the increasingly frequent exchanges and interactions between cities, the concept of resilience needs to be applied on multiple scales, such as communities, cities, and regions, and cross-city transportation network resilience research, which has become an inevitable trend [7-9]. Related research studies on the formation mechanism of crosscity transportation network resilience can enhance the selfrecovery ability of network nodes and links in the cross-city transportation network, which is beneficial for cities to mobilize resources from the network level during emergencies and reduce losses and quickly recover after disturbance occurring [10-12]. Therefore, the resilience management of cross-city transportation network has gradually become a new research paradigm.

Existing research studies on resilience management of cross-city transportation networks have found that local disturbances faced by regional transportation network will be amplified and become common disturbances by crosscity transportation $[3,13]$. Due to the difficulty of data acquisition, the research object of cross-city transportation network resilience management is mainly a single category of cross-city transportation, such as railway network and highway network [14-18]. Some studies have studied the 
resilience of different cross-city transportation networks from a static perspective, such as air transportation network [19-21] and water transportation networks [22-24]. However, most of the related research studies focus on the assessment and comparative analysis of resilience for different types of cross-city transportation networks [14, 15, 22, 25]. Research contents of previous research studies are mainly based on the assessment methods of cross-city transportation network resilience [26]. The formation mechanism of cross-city transportation network resilience has not received enough attention in previous research studies $[13,27]$. There is a lack of research studies on the optimization and improvement of cross-city transportation network resilience from a system perspective [28, 29].

Besides, few research studies on the formation mechanism of the cross-city transportation network resilience focus on the changing of network scale and structure influencing cross-city transportation network resilience $[22,30,31]$. For example, Miller-Hooks et al. used the Monte Carlo simulation method to measure the resilience of the American railroad network and found that the resilience can be improved by optimizing the topological structure of the transportation network and other intrinsic properties [32]. Ip and Wang constructed a China railway network with cities as nodes and railways as links, and the results showed that city nodes with higher resilience were mainly located in the core part of the network [30]. Osei-Asamoah and and Lownes used simulation methods to study the resilience of the railroad network in Connecticut and Indiana, and the analysis results pointed out that the railroad network exhibited biological network characteristics and had the resilience properties such as redundancy and robustness [16]. Bhatia et al. also used simulation methods to study the impact of the 2004 Indian Ocean tsunami and the 2012 North India blackout on the resilience of the Indian railway network, and the quantitative analysis results showed that the improvement of network centrality could improve the recovery speed and efficiency of railway network after disasters [31]. Ouyang Min analyzed the resilience of the China railway network from the perspective of vulnerability and found that the weighted shortest path model was more suitable for the resilience analysis of the China railway network [17].

In general, the existing research studies on the formation mechanism of cross-city transportation network resilience were limited to qualitative theoretical descriptions. The connotation of cross-city transportation network resilience was not clear, and existing research studies also did not establish a quantitative model for analyzing the cross-city transportation network resilience considering the network structure characteristics. Thus, it is necessary to analyze the formation mechanism of cross-city transportation network resilience from a systematic perspective.

Based on the practical needs of cross-city transportation network resilience management, this study takes cross-city transportation network resilience as the research object and aims to reveal the formation mechanism of cross-city transportation network resilience by complex network analysis methods. The following research questions will be answered in this study. (1) What is the connotation of crosscity transportation network resilience? (2) How is cross-city transportation network resilience formed? (3) How to theoretically describe and solve cross-city transportation network resilience?

The remainder of this paper is structured as follows. Section 2 describes the connotation of cross-city transportation network resilience considering the specific response processes of the cross-city transportation network to external disturbances. Section 3 analyzes the formation process and path of cross-city transportation network resilience. In Section 4, the theoretical model of cross-city transportation network resilience is constructed and solved. China railway network is selected as a case study in Section 5 . Finally, Section 6 summarizes the research contents of this study and explores future research directions.

\section{Connotation of Cross-City Transportation Network Resilience}

Resilience comes from the Latin word "resilio," its original meaning is "to jump back" $[33,34]$. The initial definition of resilience refers to the ability of a material to absorb energy when elastically deformed and release energy when unloaded $[35,36]$. The first definition of resilience was proposed by Holling in the field of social ecosystems in 1973, which focused on the ability of the system to absorb and adapt to various unexpected disturbances in the future through system design [37]. The widely accepted definition of resilience is the ability of the system to recover from an unfavorable state to a normal state or adjust itself to a new state according to new needs or circumstances. Thus, resilience reflects the adaptability and survivability of the system [38]. Generally, resilience is still a relatively new concept. Different disciplines have different cognitions and understandings, and the connotation of resilience is still continuously developed and improved in the development process of resilience science.

A cross-city transportation network is constituted by nodes and links which are cities and transportation infrastructure between cities, respectively. The nodes and links of cross-city transportation network present an evolution process, and the coupling relationship between the nodes and links of cross-city transportation network shows dynamic characteristics $[2,39]$. The system performance of a cross-city transportation network can be calculated by the cumulative degree of nodes. Thus, the system performance of cross-city transportation network reflects the maximum actual transportation demand that can be met between different cites, which determines the impact of cross-city transportation network on human social and economic activities [40, 41]. Based on the dynamic evolution characteristics of the cross-city transportation network, crosscity transportation network resilience is defined in this study as cross-city transportation network adopts its network topology and evolution characteristics to resist external disturbances and maintain a certain system performance and quickly restore the ability to balance after the disturbance disappears. According to the above definition of cross- 
city transportation network resilience, this study divides the conceptual connotation of cross-city transportation network resilience into three different stages: resistance stage, absorption stage, and recovery stage, which are shown in Figure 1.

Figure 1 describes the three stages of cross-city transportation network resilience. Before disturbances occur, the system performance of the cross-city transportation network is in an equilibrium state. After disturbances occur, crosscity transportation network resilience is firstly reflected by the resistance ability of cross-city transportation networks to external disturbances. When cross-city transportation network can resist external disturbances, cross-city transportation network maintains original equilibrium state, or external disturbances have a negligible impact on the system performance of cross-city transportation network. When cross-city transportation network cannot resist external disturbances, cross-city transportation network resilience is reflected by the absorption ability of cross-city transportation network to external disturbances. At the absorption stage, the cross-city transportation network can maintain a certain system performance to meet the basic transportation needs between cities. After disturbances disappear, cross-city transportation network resilience is reflected by the system performance recovery of the crosscity transportation network. At the recovery stage, the system performance of the cross-city transportation network is recovered to its original equilibrium state, or a new equilibrium state by adjusting its network characteristics to adapt to the external environment, which reflects the adaptive characteristics of the cross-city transportation network.

\section{Formation Process and Path of Cross-City Transportation Network Resilience}

3.1. Formation Process of Cross-City Transportation Network Resilience. By defining the connotation of cross-city transportation network resilience in Section 2, the formation process of cross-city transportation network resilience is divided into three stages: resistance stage, absorption stage, and recovery stage. Before disturbances occur, the cross-city transportation network is in a static equilibrium state, and its network state is described by static model SM:

$$
\mathrm{SM}=\operatorname{SM}(N, L, d)
$$

The static model of the cross-city transportation network in equation (1) includes three types of elements: nodes, links, and degree. In equation (1), $N=\left\{n_{1}, n_{2}, \ldots, n_{i}\right\}$ is the set of city nodes in cross-city transportation network and $L=\left\{l_{1}, l_{2}, \ldots, l_{j}\right\}$ is the set of transportation infrastructure links between different city nodes in cross-city transportation network. The degree of link $l_{i} \in L$ is $d_{i}$; $d=\left\{d_{1}, d_{2}, \ldots, d_{j}\right\}$ is the set of all degree $d_{i}$ in the cross-city transportation network. The degree of link $l_{i}$ is $d_{i}$, which is determined by the number of transportation infrastructure links between two city nodes. The static model of the cross-

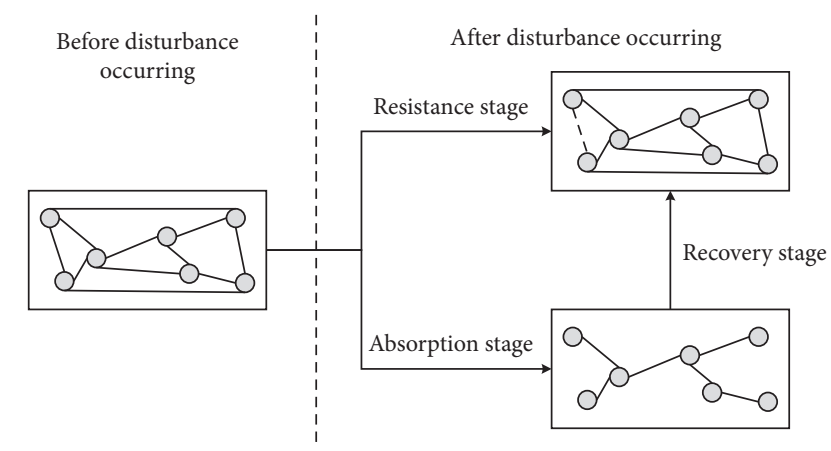

FIgURE 1: Stages division of cross-city transportation network resilience.

city transportation network defines links between nodes as undirected links:

$$
l=l\left(n_{a}, n_{b}\right)=l\left(n_{b}, n_{a}\right) .
$$

In equation (2), $n_{a}$ and $n_{b}$ are the starting point (ending point) and ending point (starting point) of link $l$, respectively, where $n_{a}, n_{b} \in N$, and $n_{a} \neq n_{b}$.

When cross-city transportation network can resist external disturbances, cross-city transportation network maintains the original equilibrium state or the external disturbances have negligible impacts on the system performance of cross-city transportation network. The network state of the cross-city transportation network is still described by equation (1). When cross-city transportation network cannot resist external disturbances, cross-city transportation network resilience is reflected by the absorption ability of cross-city transportation network to external disturbances. At the absorption stage, the cross-city transportation network can maintain a certain system performance to meet the basic transportation needs between cities, and the network state of the cross-city transportation network is described by the dynamic model SDM:

$$
\operatorname{SDM}=\operatorname{SDM}(N(t), L(t), d(t)) .
$$

In equation (3), node $N$ and link $L$ in the cross-city transportation network show dynamic evolution characteristics over time, where $N(t)$ and $L(t)$ are the numbers of city nodes and the number of links between city nodes in cross-city transportation network at time $t$, respectively. The changes of city nodes $N(t)$ and links $L(t)$ between city nodes will lead to the dynamic evolution of cross-city transportation network structure. In general, the change of city nodes $N(t)$ and links $L(t)$ between city nodes will cause a change in degree $d$ of the cross-city transportation network, which means that the degree of cross-city transportation network will change from $d$ to $d(t)$. Dynamic model $S D M$ describes the dynamic changes of network structure and degree of the cross-city transportation network, which reflects the random characteristics of the cross-city transportation network and the changes of the transportation state. Therefore, the dynamic model S DM explains the dynamic evolution characteristics of the cross-city transportation network from a macroperspective. 
After disturbances disappear, cross-city transportation network resilience is reflected by the system performance recovery of the cross-city transportation network. At the recovery stage, the system performance of the cross-city transportation network is recovered to its original equilibrium state or a new equilibrium state by adjusting the network characteristics to adapt to the external environment. At the recovery stage, the state of the cross-city transportation network is described by new static model $\mathrm{SM}_{n}$ :

$$
\mathrm{SM}_{n}=\operatorname{SM}_{n}\left(N_{n}, L_{n}, d_{n}\right) .
$$

In equation (4), $N_{n}$ is the set of city nodes in cross-city transportation network after the recovery stage is completed, $L_{n}$ is the set of links between different city nodes in cross-city transportation network after the recovery stage is completed, and $d_{n}$ is the set of all degrees in the cross-city transportation network after the recovery stage is completed. In the formation process of cross-city transportation network resilience, the logical relationship between the three stages is summarized in Figure 2.

\subsection{Formation Path of Cross-City Transportation Network} Resilience. The formation process of cross-city transportation network resilience can be divided into resistance stage, absorption stage, and recovery stage, which form different resilience characteristics at different stages. After disturbances occur, cross-city transportation network firstly resists the impacts of external disturbances [42, 43]. When cross-city transportation network cannot resist external disturbances, cross-city transportation network absorbs the impacts of external disturbances [44, 45]. After disturbances disappear, the cross-city transportation network recovers its system performance $[46,47]$. The above three stages, respectively, reflect the resistance ability, absorption ability, and recovery ability of the cross-city transportation network to external disturbances. Therefore, cross-city transportation network resilience has different formation paths at different stages, as shown in Figure 3.

Figure 3 summarizes the formation path of cross-city transportation network resilience. Different formation paths are divided by the time points of the horizontal axis and labeled with different line types. Point $b$ in Figure 3 is the threshold point of external disturbances impacts on the cross-city transportation network. Before point $b$, cross-city transportation network statically resists external disturbances. After point $b$, the cross-city transportation network dynamically absorbs external disturbances and recovers to a balanced state after disturbances disappear. Therefore, the formation paths of cross-city transportation network resilience are divided into static formation path and dynamic formation path. In the static formation path, cross-city transportation network resilience is formed by the static resistance of city nodes to external disturbance, which reflects the nodes' resilience of the cross-city transportation network. In the dynamic formation path, cross-city transportation network resilience is formed by the dynamic absorption and recovery of the network system to external

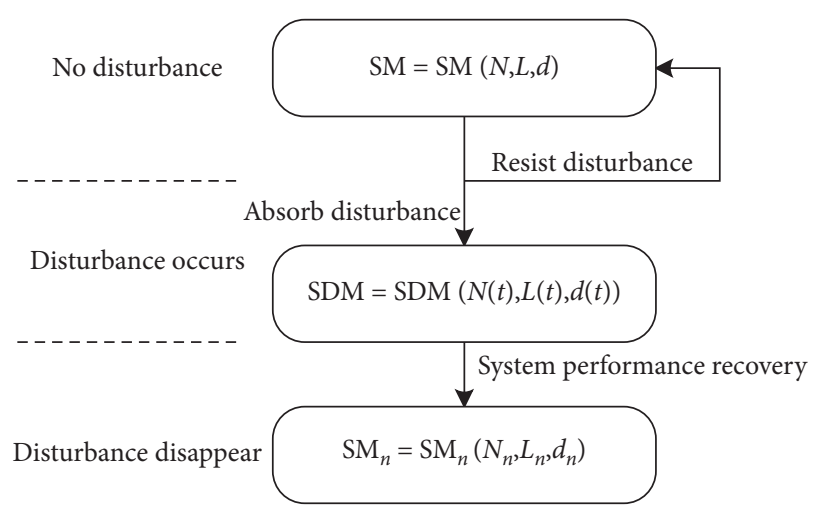

FIgURE 2: Logical relationship between three stages.

disturbances, which reflects the system resilience of crosscity transportation network.

\subsubsection{Static Formation Path of Cross-City Transportation} Network Resilience. The static formation path is the solid line $a b$ in Figure 3, and cross-city transportation network resilience reflects the static resistance of city nodes to external disturbances. This study defines $\left[t_{0}, t_{1}\right)$ period as the resistance stage. At the resistance stage, the external disturbance faced by the cross-city transportation network is less than or equal to the resistance ability of the cross-city transportation network. Thus, the cross-city transportation network maintains the original static equilibrium state, and its state is described by the static model $\mathrm{SM}=\operatorname{SM}(N, L, d)$. At the resistance stage, network nodes' resilience is reflected by the resistance ability of city nodes to external disturbances, which is essentially the resilience of cities in the cross-city transportation network to external disturbances [42].

Point $b$ is the threshold point of external disturbances impact on the cross-city transportation network, and static formation path may be transformed into a dynamic formation path at point $b$. If cross-city transportation network can still resist external disturbances in period $\left[t_{1}, t 4\right]$, which means that external disturbance is less than or equal to the resistance ability of city nodes in the cross-city transportation network, then cross-city transportation network resilience will be manifested as the static resistance of the city nodes to external disturbances in period $\left[t_{1}, t 4\right]$. The static formation path will not be converted into a dynamic formation path, as shown by the extended broken line of the solid line $a b$ in Figure 3. When the external disturbance is less than or equal to the resilience resistance of city nodes in cross-city transportation network, cross-city transportation network resilience is manifested as the static resistance path of city nodes to the external disturbance in the period $\left[t_{1}, t 4\right]$.

If cross-city transportation network cannot continue to resist external disturbances at point $b$, which means that external disturbance is larger than the resistance ability of city nodes in the cross-city transportation network. Then, the static formation path will be transformed into a dynamic formation path at point $b$, which is shown by the broken line 


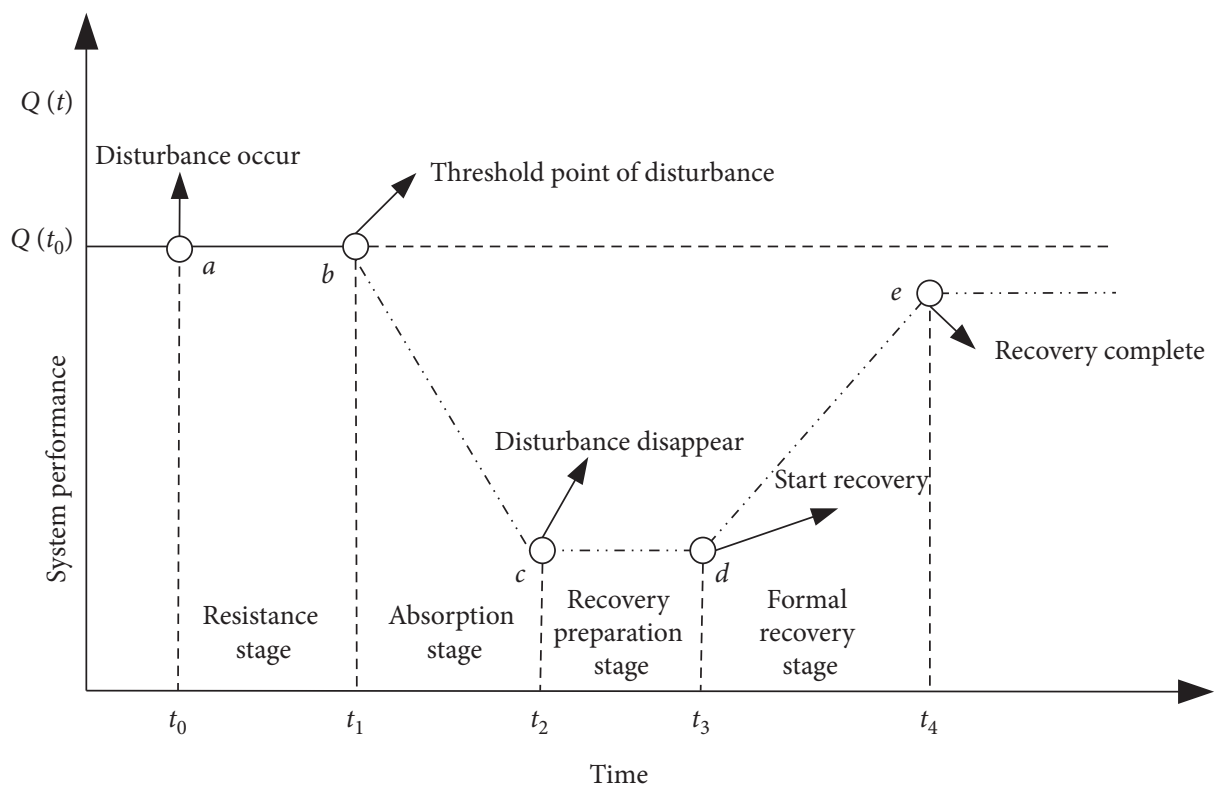

FIGURE 3: Formation path of cross-city transportation network resilience.

$b c$ de in Figure 3. Dynamic formation path is the dynamic continuation of static formation path at the threshold pointb, which reflects the system resilience of cross-city transportation network.

\subsubsection{Dynamic Formation Path of Cross-City Transportation} Network Resilience. When an external disturbance at the threshold point $b$ is larger than the resistance ability of crosscity transportation network, cross-city transportation network resilience will change from a static formation path at resistance stage to a dynamic formation path. Disturbance disappearing point $b$ divides the dynamic formation path into the absorption stage and recovery stage. As shown in Figure 3, the absorption stage is the dashed line $b c$ in period $\left[t_{1}, t_{2}\right]$, and the recovery stage is the broken line cde in the period $\left(t_{2}, t_{4}\right]$.

In dynamic formation path, cross-city transportation network resilience is firstly reflected by the dynamic absorption ability of cross-city transportation network to external disturbance. At the absorption stage, the network state of the cross-city transportation network changes under the external disturbance. The absorption ability of the crosscity transportation network is finally manifested as the network performance level that can be maintained when external disturbance disappears. At the absorption stage, cross-city transportation network resilience reflects the network performance loss $Q_{L}$ of the cross-city transportation network, $Q_{L}=Q\left(t_{0}\right)-Q\left(t_{2}\right)$, as shown in Figure 3.

After external disturbance disappears at point $c$, the dynamic formation path of cross-city transportation network resilience is reflected by the dynamic recovery of system performance in period $\left(t_{2}, t_{4}\right]$, which means dynamic formation path transfer to the recovery stage. The recovery stage can be further divided into the recovery preparation stage and the formal recovery stage. As shown in Figure 3, the dynamic formation path of the recovery preparation stage is described by the dotted line cd in period $\left(t_{2}, t_{3}\right)$. The dynamic formation path of the formal recovery stage is described by the dotted line de in period $\left[t_{3}, t_{4}\right]$. Different recovery plans are selected at the recovery preparation stage, and the final recovery plan is determined as the dynamic path point $d$ at time $t_{3}$. At the formal recovery stage, the system performance of cross-city transportation network recovers in period $\left[t_{3}, t_{4}\right]$. The system performance of crosscity transportation network recovers along the dynamic formation path dotted line de in Figure 3 and returns to a new equilibrium state $Q\left(t_{4}\right)$ at time $t_{4}$.

\section{Theoretical Model of Cross-City Transportation Network Resilience}

This study divides the formation process of cross-city transportation network resilience into resistance stage, absorption stage, and recovery stage. At different stages, the cross-city transportation network shows different resilience characteristics. Based on the analysis results of the formation process and formation path of cross-city transportation network resilience, the standard linear solid model $[44,46,48,49]$ is used to qualitatively describe the formation process of cross-city transportation network resilience. The qualitative model of cross-city transportation network resilience is summarized in Figure 4.

Figure 4 is the qualitative model of cross-city transportation network resilience. The left side of the qualitative model is the external disturbance input $D(t)$, and the right side is the internal constraint boundary of the cross-city transportation network. The qualitative model consists of two series units, namely, resistance unit $C_{1}$ and absorption unit $C_{2}$. Resistance unit $C_{1}$ is an elastic unit, which works in the process of cross-city transportation network resisting 


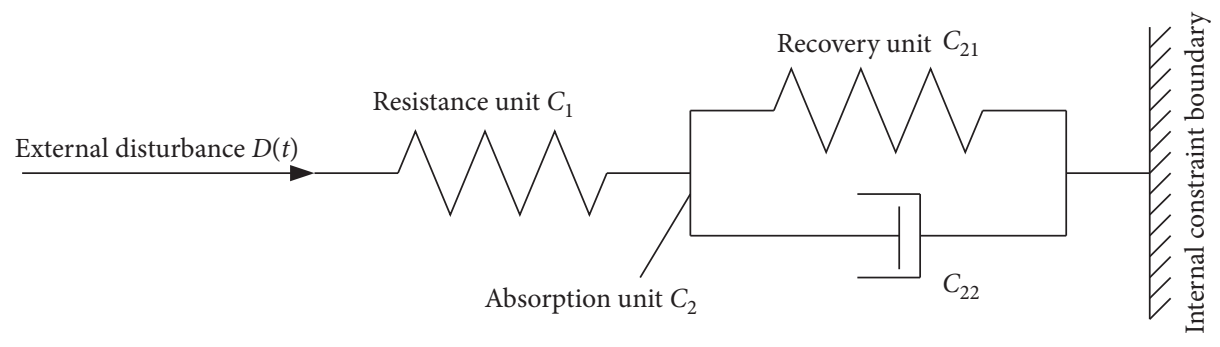

FIgURE 4: Qualitative model of cross-city transportation network resilience.

external disturbance $D(t)$. When resistance unit $C_{1}$ cannot resist external disturbance $D(t)$, the absorption unit $C_{2}$ begin to work. Absorption unit $C_{2}$ is composed of two parallel subunits $C_{21}$ and $C_{22}$, where the subunit $C_{21}$ is an elastic unit and the subunit $C_{22}$ is a damping unit. The subunits $C_{21}$ and $C_{22}$ jointly absorb the impacts of external disturbance $D(t)$ on the system performance of cross-city transportation network. When external disturbance $D(t)$ disappears, subunit $C_{21}$ in the absorption unit $C_{2}$ is converted into a recovery unit, and the recovery unit $C_{21}$ restores cross-city transportation network to a new equilibrium state. The system performance $Q(t)$ of the crosscity transportation network is determined by absorption unit $C_{2}$, and resistance unit $C_{1}$ does not affect the system performance $Q(t)$ of the cross-city transportation network.

4.1. Theoretical Model Construction. External disturbance $D(t)$ has the same influence on all parts of the cross-city transportation network, which means that the external disturbance influence function received by the resistance unit $C_{1}$ and the absorption unit $C_{2}$ is the same. The influence functions of external disturbance $D(t)$ on the resistance unit $C_{1}$ and the absorption unit $C_{2}$ are described as follows:

$$
D_{1}(t)=D_{2}(t)=D(t)
$$

In equation (5), $D_{1}(t)$ and $D_{2}(t)$ are the influence functions of external disturbance $D(t)$ on absorption unit $C_{1}$ and resistance unit $C_{2}$, respectively. At the resistance stage, external disturbance $D(t)$ firstly influences the resistance unit $C_{1}$, and the performance of resistance unit $C_{1}$ produces a loss $\varepsilon_{1}(t)$. When external disturbance $D(t)$ is less than or equal to the resistance ability of cross-city transportation network, the performance loss $\varepsilon_{1}(t)$ of resistance unit $C_{1}$ is within the elastic limit of the resistance unit $C_{1}$. Thus, the resistance unit $C_{1}$ can completely resist the negative impact of external disturbance $D(t)$ on the system performance of cross-city transportation network. The performance loss $\varepsilon_{1}(t)$ of resistance unit $C_{1}$ can be calculated by equation (6).

$$
\varepsilon_{1}(t)=\frac{D_{1}(t)}{k_{1}} .
$$

In equation (6), $k_{1}$ is the elastic coefficient of the resistance unit $C_{1}$ which reflects the resistance ability dimension of the cross-city transportation network to external disturbance $D(t)$. When external disturbance $D(t)$ is larger than the resistance ability of cross-city transportation network, the performance loss $\varepsilon_{1}(t)$ reaches the elastic limit of the resistance unit $C_{1}$. Then, the cross-city transportation network enters the absorption to external disturbance $D(t)$.

At the absorption stage, the absorption unit $C_{2}$ is composed of two parallel subunits $C_{21}$ and $C_{22}$. The absorption unit $C_{2}$ and two parallel subunits $C_{21}$ and $C_{22}$ have the same external disturbance influence function, which are $D_{2}(t), D_{21}(t)$, and $D_{22}(t)$, respectively. The absorption unit $C_{2}$ decomposes the performance loss $\varepsilon_{2}(t)$ caused by external disturbance influence function $D_{2}(t)$ into two parallel subunits $C_{21}$ and $C_{22}$, which are named as $\varepsilon_{21}(t)$ and $\varepsilon_{22}(t)$, respectively. At the absorption stage, the relationship between external disturbance influence function and performance loss of absorption unit $C_{2}$, subunits $C_{21}$ and $C_{22}$, is shown in equations (7) and (8):

$$
\begin{gathered}
D_{2}(t)=D_{21}(t)=D_{22}(t), \\
\varepsilon_{2}(t)=\varepsilon_{21}(t)+\varepsilon_{22}(t) .
\end{gathered}
$$

The subunit $C_{21}$ is an elastic unit, and its elastic coefficient is $k_{2}$. Under the action of external disturbance $D_{21}(t)$, the performance loss $\varepsilon_{21}(t)$ of subunit $C_{21}$ can be calculated by the following equation:

$$
\varepsilon_{21}(t)=\frac{D_{21}(t)}{k_{2}} .
$$

The subunit $C_{22}$ is a damping unit, and its absorption function to external disturbances is a cumulative process. The performance loss $\varepsilon_{22}(t)$ is affected by external disturbance $D_{22}(t)$ and damping coefficient $\mu$, which can be calculated by the following equation:

$$
\varepsilon_{22}(t)=\int_{t_{1}}^{t} \frac{D_{22}(t)}{\mu} \mathrm{d} t .
$$

In equation (10), $t_{1}$ is the time corresponding to the external disturbance threshold point in Figure 3. The elastic coefficient $k_{2}$ of subunit $C_{21}$ and the damping coefficient $\mu$ of subunit $C_{22}$ jointly determine the absorption capacity of the absorbing unit $C_{2}$ to external disturbance $D_{2}(t)$. According to equations (7)-(10), the performance loss $\varepsilon_{2}(t)$ of absorption unit $C_{2}$ can be calculated by external disturbance influence function $D_{2}(t)$, the elastic coefficient $k_{2}$ of subunit $C_{21}$, and the damping coefficient $\mu$ of subunit $C_{22}$ together: 


$$
\varepsilon_{2}(t)=\frac{D_{2}(t)}{k_{2}}+\int_{t_{1}}^{t} \frac{D_{2}(t)}{\mu} \mathrm{d} t .
$$

When external disturbance $D(t)$ disappears at time $t_{2}$, cross-city transportation network enters the recovery preparation stage. At the recovery preparation stage, the system performance of cross-city transportation network will be kept unchanged and the system performance of cross-city transportation network is $Q\left(t_{2}\right)$. Different recovery plans are compared at the recovery preparation stage, and the final recovery plan is determined at time $t_{3}$. At time $t_{3}$, cross-city transportation network enters the formal recovery stage.

At the formal recovery stage, the subunit $C_{21}$ of absorption unit $C_{2}$ is converted into a recovery unit and the performance loss of subunit $C_{21}$ at the absorption stage is recovered. At time $t_{4}$, the system performance of the crosscity transportation network is recovered to a new equilibrium state. Recovery unit $C_{21}$ recovers to its original performance and the final recovery performance is $\varepsilon_{21}^{r}$ which can be determined by

$$
\varepsilon_{21}^{r}=\frac{D_{2}\left(t_{2}\right)}{k_{2}}
$$

4.2. Theoretical Model Solution. The theoretical model of cross-city transportation network resilience indicates that the system performance of the cross-city transportation network shows different evolution trends at different stages. Thus, the theoretical model of cross-city transportation network resilience needs to be solved in different stages. At the resistance stage, cross-city transportation network can completely resist the influence of external disturbance $D(t)$ on the system performance $Q(t)$ of cross-city transportation network through resistance unit $C_{1}$, and the system performance of cross-city transportation network remains as the initial performance $Q\left(t_{0}\right)$. External disturbance $D(t)$ reaches threshold point $b$ at time $t_{1}$, and cross-city transportation network enters the absorption stage to external disturbance $D(t)$. During the period $\left[t_{1}, t_{2}\right]$, the system performance $Q(t)$ of cross-city transportation network gradually decreases under the action of external disturbance $D(t)$ :

$$
Q(t)=Q\left(t_{0}\right)-\varepsilon_{2}(t) .
$$

In equation (13), $\varepsilon_{2}$ is the real-time system performance loss of cross-city transportation network at absorption stage, which can be calculated by equation (11). External disturbance $D(t)$ disappears at $t_{2}$, and cross-city transportation network enters the recovery preparation stage. The system performance of cross-city transportation network is kept as $Q\left(t_{2}\right)$ at the recovery preparation stage. According to equation (13), $Q\left(t_{2}\right)$ can be described as

$$
Q\left(t_{2}\right)=Q\left(t_{0}\right)-\varepsilon_{2}\left(t_{2}\right) .
$$

Cross-city transportation network enters the formal recovery stage at time $t_{3}$, and the system performance of cross-city transportation network recovers to a new equilibrium state at $t_{4}$ through the performance recovery of the recovery unit $C_{21}$. This study assumes that the system performance of the cross-city transportation network shows linear recovery characteristics at the formal recovery stage. The system performance of cross-city transportation network increases by $\varepsilon_{21}^{r}$ at time $t_{4}$ compared with the system performance at time $t_{3}$. Thus, the system performance $Q\left(t_{4}\right)$ of cross-city transportation network at time $t_{4}$ is described as

$$
Q\left(t_{4}\right)=Q\left(t_{0}\right)-\varepsilon_{2}\left(t_{2}\right)+\varepsilon_{21}^{r} .
$$

Through the above analysis, the system performance of the cross-city transportation network at different stages can be obtained. The system performance of cross-city transportation network $Q(t)$ at each stage is shown as

$$
Q(t)= \begin{cases}Q\left(t_{0}\right), & t_{0} \leq t<t_{1}, \\ Q\left(t_{0}\right)-\varepsilon_{2}(t), & t_{1} \leq t \leq t_{2}, \\ Q\left(t_{0}\right)-\varepsilon_{2}\left(t_{2}\right), & t_{2}<t<t_{3}, \\ Q\left(t_{0}\right)-\varepsilon_{2}\left(t_{2}\right)+\frac{\varepsilon_{21}^{r}}{\left(t_{4}-t_{3}\right)}\left(t-t_{3}\right), & t_{3} \leq t \leq t_{4} .\end{cases}
$$

Equations (5), (11), and (12) are incorporated into equation (16), and the final quantified system performance of cross-city transportation network under external disturbance $D(t)$ at each stage is shown as

$$
Q(t)= \begin{cases}Q\left(t_{0}\right), & t_{0} \leq t<t_{1}, \\ Q\left(t_{0}\right)-\frac{D(t)}{k_{2}}-\int_{t_{1}}^{t} \frac{D(t)}{\mu} \mathrm{d} t, & t_{1} \leq t \leq t_{2}, \\ Q\left(t_{0}\right)-\frac{D\left(t_{2}\right)}{k_{2}}-\int_{t_{1}}^{t_{2}} \frac{D(t)}{\mu} \mathrm{d} t, & t_{2}<t<t_{3}, \\ Q\left(t_{0}\right)-\frac{D\left(t_{2}\right)}{k_{2}}-\int_{t_{1}}^{t_{2}} \frac{D(t)}{\mu} \mathrm{d} t+\frac{D\left(t_{2}\right)}{\left(t_{4}-t_{3}\right) k_{2}}\left(t-t_{3}\right), & t_{3} \leq t \leq t_{4} .\end{cases}
$$




\section{Case Study of China Railway Network Resilience}

5.1. China Railway Network Resilience Construction. As a typical cross-city transportation network, China railway network nodes represent different cities and links manifest the railway links between cities. In this section, China railway network is selected as a case study for testing formation mechanism of cross-city transportation network resilience proposed in this study. China railway network is constructed according to the following rules [13, 50]:

(1) Network nodes: network nodes are prefecture-level cities that have railway passenger access.

(2) Network links: links are the railway links between different prefecture-level cities.

(3) Weighted network: the degree of one city node is defined as the accumulative number of links from this city to the other cities of China railway network.

(4) Undirected network: not all railway links between the different cities of China railway network appear in pairs. Thus, the degrees between two cities are not completely symmetrical. Here, each network is simplified as an undirected network.

This study constructs China railway network in 1957, 1985, 1987, 1997, 1998, 2000, 2007, 2008, 2009, 2014, 2015, 2016, and 2017, respectively. Most prefecture-level cities have been connected to China railway network after 2017, and the scale characteristics of China railway network between 2018 and 2020 are consistent compared with 2017. Thus, the data of China railway network from 2018 to 2020 are not collected for analyzing the resilience characteristics of China railway network. Table 1 summarizes the scale characteristic indicators of the China railway network.

As shown in Table 1, the China railway network presents dynamic evolution characteristics. The network scale of the China railway network is getting larger and larger, which is manifested by the increase of city nodes number and degree strength between city nodes. According to the analysis results on the formation process and path of cross-city transportation network resilience in Section 3, the dynamic evolution of the China railway network scale will lead to corresponding evolution on China railway network nodes' resilience and system resilience. Thus, this section selects the above China railway network as the research object, and the theoretical model of cross-city transportation network resilience established in Section 4 is used to conduct a case study for analyzing the evolution characteristics of China railway network nodes' resilience and system resilience.

5.2. Evolution Characteristics of Nodes' Resilience. This section selects 96 city nodes that have always existed in the China railway network as the research object, and the evolution characteristics of these 96 city nodes resilience are analyzed in 13 years. The evolution characteristics of China railway network nodes' resilience essentially reveal the evolution characteristics of city resistance ability to external
TABLE 1: Scale characteristic indicators of China railway network.

\begin{tabular}{cccc}
\hline Year & $N$ & $D$ & AD \\
\hline 1957 & 114 & 8560 & 75 \\
1985 & 164 & 14166 & 86 \\
1987 & 174 & 17760 & 102 \\
1997 & 219 & 32626 & 149 \\
1998 & 216 & 35684 & 165 \\
2000 & 230 & 55230 & 240 \\
2007 & 242 & 70724 & 292 \\
2008 & 243 & 77440 & 319 \\
2009 & 245 & 88314 & 360 \\
2014 & 268 & 133633 & 499 \\
2015 & 269 & 268047 & 996 \\
2016 & 273 & 288823 & 1058 \\
2017 & 274 & 300946 & 1098 \\
\hline
\end{tabular}

Note. $N$ is nodes' number; $D$ is degree; $\mathrm{AD}=D / N$, which is the average degree.

disturbances in the China railway network. The evolution characteristics of 96 city nodes' resilience in the China railway network are calculated by the theoretical model proposed in Section 4 and shown in Figure 5.

Figure 5 summarizes the evolution characteristics of 96 city nodes' resilience in the China railway network. The point line is the evolution curve of 96 city nodes' resilience, and the evolution curve of the 96 city nodes' average resilience is described by the thick black line. Figure 5 shows that 96 city nodes' resilience in the China railway network generally increases over time, but the evolution characteristics of 96 city nodes' resilience show certain differences in different time intervals. According to the differences of city nodes' resilience in different time intervals, the period from 1957 to 2017 is divided into four evolution stages: (1) volatile growth stage (1957-1987), city nodes' resilience showed volatility with time, (2) gentle growth stage (1987-2009), city nodes' resilience increased slowly with time, (3) rapid growth stage (2009-2014), city nodes' resilience increased rapidly over time, and (4) stable stage (2014-2017), city nodes' resilience tended to stabilize over time.

As shown in Figure 5, the resilience values of most city nodes are greater than the average value of 96 city nodes' resilience. The resilience values of few city nodes are significantly lower than the average value of 96 city nodes' resilience, which results in the resilience values of 96 city nodes at a relatively low level. Although the resilience value of different city nodes present differences, these differences have gradually decreased over time and have been in a convergence trend after 2014. From 1987 to 2014, there are some city nodes, such as Beijing and Zhengzhou, the resilience values of these city nodes are significantly higher than that of other city nodes. Except for very few city nodes, such as Jixi and Yichun, the differences of resilience values between city nodes have converged and stabilized after 2014 . The above analysis results show that the opening of highspeed railways after 2007 has reduced differences in the resilience values of different city nodes. City node Jixi does not open high-speed rails from 2014 to 2017, and city node Yichun has a small number of high-speed rails from 2014 to 


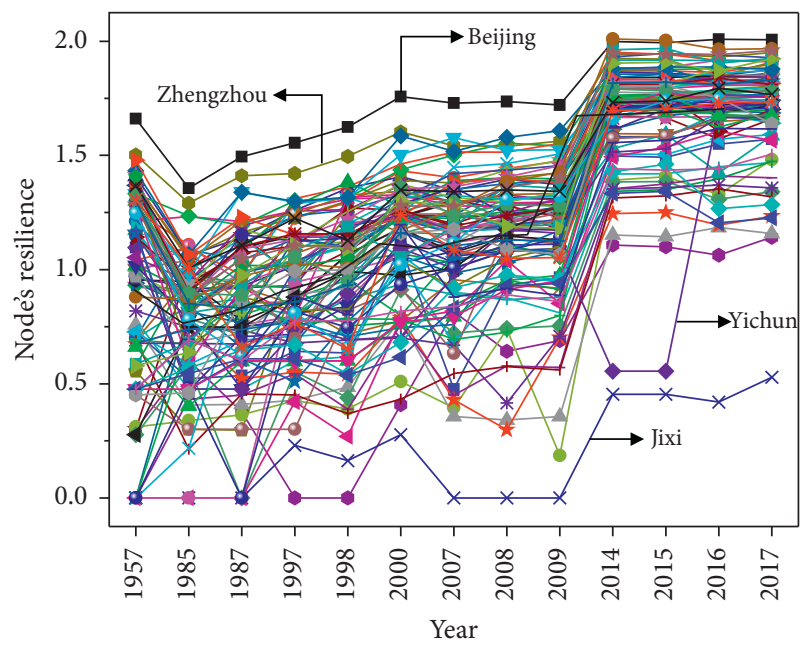

FIGURE 5: Evolution characteristics of city node resilience.

2015. Thus, the resilience values of Jixi and Yichun are still significantly lower than other 94 city nodes.

5.3. Evolution Characteristics of System Resilience. The system resilience of the China railway network is the cumulative result of absorption ability and recovery ability at the absorption stage and recovery stage. According to the analysis results on the formation process and path of crosscity transportation network resilience, the China railway network will compare and select different recovery plans at the recovery preparation stage, which is affected by the network characteristics of the China railway network. This section uses the theoretical model of cross-city transportation network resilience to quantify the formation path of China railway network system resilience in different years, which is shown in Figure 6.

Figure 6 describes the formation path curve of China railway network system resilience in different years. The decreasing curves on the left side of the formation path curve reflects the absorption path of China railway network system resilience to external disturbances, and the absorption path ends at the common threshold point of different absorption scenarios. The horizontal straight lines in the middle of the formation path curve reflect the time length of the recovery preparation stage. This study simplifies the time length of the recovery preparation stage as 10 -time units. The incremental curves on the right side of the formation path curve reflect the recovery path of China railway network system resilience, and the recovery path ends at the common threshold point of recovery behavior iterative ending under different recovery scenarios. The quantitative results of the enclosed area size of the formation path curve are shown in Figure 7.

The enclosed area size of the formation path curve in Figure 7 presents the dimension of China railway network system resilience. The larger enclosed area size of the formation path curve reflects that the China railway network presents lower system resilience to external disturbances. In contrast, the smaller enclosed area size of the formation path curve reflects that the China railway network presents higher

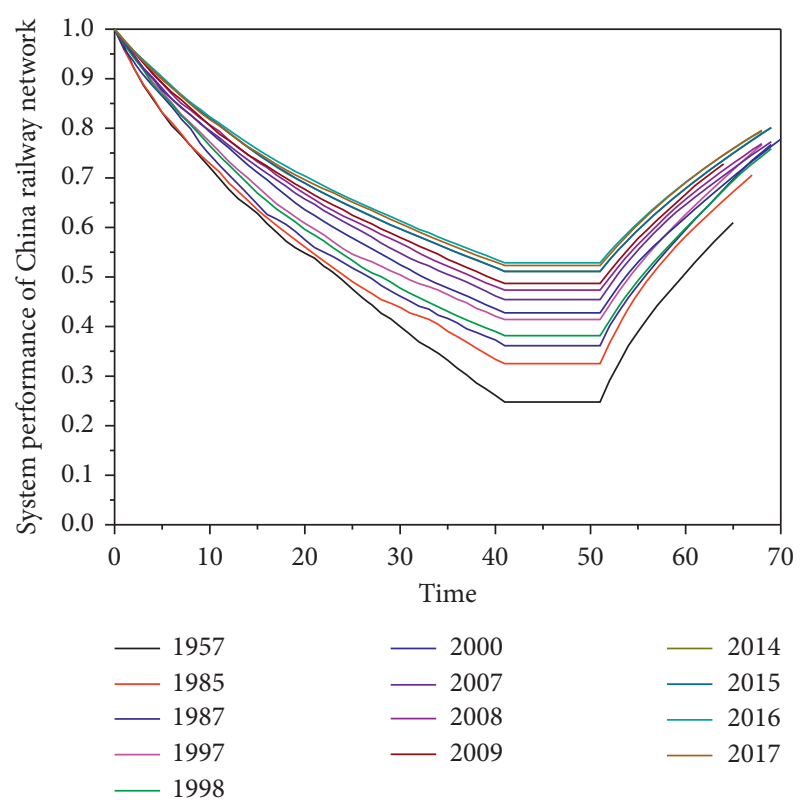

FIgURE 6: Formation path of China railway network system resilience.

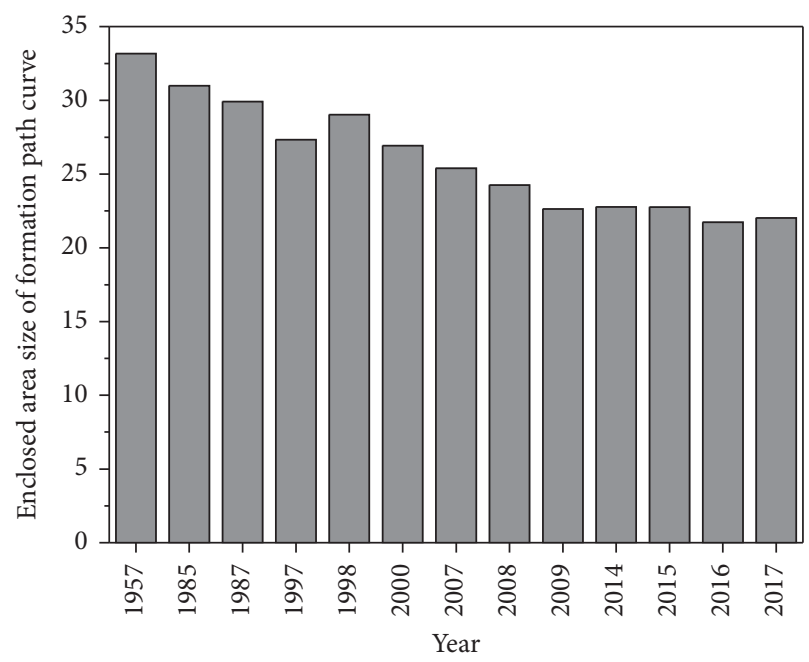

FIGURE 7: Enclosed area size of the formation path curve.

system resilience to external disturbances. As shown in Figure 7, except for 1997, the size of the formation path curve generally shows a decreasing trend overtime in the process of China railway network absorbing and recovering from external disturbances. Thus, the system resilience of the China railway network system gradually increases over time. After 2014, the number of city nodes approached the limit value of the potential city nodes' number and the system resilience of the China railway network gradually stabilized state.

\section{Conclusions}

In this study, the connotation of cross-city transportation network resilience is defined. The specific formation process 
of cross-city transportation network resilience is analyzed and summarized. Finally, a theoretical model is constructed and solved for revealing the formation mechanism of crosscity transportation network resilience. The research results show that the formation process of cross-city transportation network resilience includes three stages: resisting disturbance, absorbing disturbance, and function recovery. In the process of resisting disturbance, absorbing disturbance, and recovering functions, cross-city transportation network adjusts the network scale and structural characteristics to adapt to external disturbances and achieve a new equilibrium state, which reflects the connotation of cross-city transportation network resilience. The formation path of cross-city transportation network resilience includes the static formation path and dynamic formation path. In the static formation path, cross-city transportation network resilience is formed by the static resistance of city nodes to external disturbance. In the dynamic formation path, crosscity transportation network resilience is formed by the dynamic absorption and recovery of the network system to external disturbances.

This study constructs and solves the theoretical model of formation mechanism for cross-city transportation network resilience, which provides a qualitative and quantitative integrated research method for the resilience management of cross-city transportation network. The research results of this study can be systematically applied to resilience management practices for the different types of cross-city transportation networks, which is very important for the improvement of cross-city transportation network resilience. In general, this study systematically reveals the formation mechanism of cross-city transportation network resilience from qualitative and quantitative perspectives, which help to enhance and improve the city nodes' resilience and system resilience of the cross-city transportation network to external disturbances. Although the research object of this study is cross-city transportation network, the theoretical model proposed in this study is also suitable for the resilience analysis of transportation network in the urban area. The research results of this study provide development strategies for promoting the collaborative planning and construction of cross-city transportation network and transportation network in the urban area from the perspective of resilience.

\section{Data Availability}

The data used to support the findings of this study are available from the corresponding author upon request.

\section{Conflicts of Interest}

The authors declare that they have no conflicts of interest.

\section{Acknowledgments}

This research was supported by the National Social Science Fund of China (nos. 18ZDA043 and 19VDL001). This work was also funded by the National Natural Science Foundation of China (NSFC) (nos. 71671053 and 71841024), Guangdong Science and Technology Program (no. 2019B101001019), Key Research and Development Project of Liaoning Province (no. 2020JH2/10100042), Key Research and Development Project of Liaoning Province (no. 84200040), Social Science Planning Foundation of Liaoning Province (no. L20BGL056), Economic and Social Development Research Foundation of Liaoning Province (no. 20211slqnkt-014), and Natural Science Foundation of Heilongjiang Province (no. LH2019G006).

\section{References}

[1] L. M. A. Bettencourt, J. Lobo, D. Helbing, C. Kuhnert, and G. B. West, "Growth, innovation, scaling, and the pace of life in cities," Proceedings of the National Academy of Sciences, vol. 104, no. 17, pp. 7301-7306, 2007.

[2] L. Wang, T Wang, M Shao et al., "The emission quenching of upconversion nanoparticles coated with amorphous silica by fluorescence resonance energy transfer: a mercury-sensing nanosensor excited by near-infrared radiation," Spectrochimica Acta. Part A, Molecular and Biomolecular Spectroscopy, vol. 254, Article ID 119608, 2021.

[3] G. S. Van Der Vegt, P. Essens, M. Wahlström, and G. George, "Managing risk and resilience," Academy of Management Journal, vol. 58, no. 4, pp. 971-980, 2015.

[4] E. Porse and J. Lund, "Network structure, complexity, and adaptation in water resource systems," Civil Engineering and Environmental Systems, vol. 32, no. 1-2, pp. 143-156, 2015.

[5] C. Mason and E. Strankalis, "Network resilience: a new way to target maintenance spending," Proceedings of the Institution of Civil Engineers-Civil Engineering, vol. 164, no. 4, p. 149, 2011.

[6] B. Genge and C. Siaterlis, "Analysis of the effects of distributed denial-of-service attacks on MPLS networks," International Journal of Critical Infrastructure Protection, vol. 6, no. 2, pp. 87-95, 2013.

[7] S. Meerow, J. P. Newell, and M. Stults, "Defining urban resilience: a review," Landscape and Urban Planning, vol. 147, pp. 38-49, 2016.

[8] L. Wang, X. Xue, Z. Wang, and L. Zhang, "A unified assessment approach for urban infrastructure sustainability and resilience," Advances in Civil Engineering, vol. 2018, Article ID 2073968, 19 pages, 2018.

[9] E. C. Portante, J. A. Kavicky, B. A. Craig, and L. E. Talaber, "Modeling electric power and natural gas system interdependencies," Journal of Infrastructure Systems, vol. 23, no. 4, Article ID 04017035, 2017.

[10] L. Wang, X. Xue, R. J. Yang, and X. Luo, "Built environment and management: exploring grand challenges and management issues in built environment," Frontiers of Engineering Management, pp. 1-14, 2019.

[11] X. Zhang, E. Miller-Hooks, and K. Denny, "Assessing the role of network topology in transportation network resilience," Journal of Transport Geography, vol. 46, pp. 35-45, 2015.

[12] R. Faturechi and E. Miller-Hooks, "Measuring the performance of transportation infrastructure systems in disasters: a comprehensive review," Journal of Infrastructure Systems, vol. 21, no. 1, Article ID 04014025, 2015.

[13] L. Wang, X. Xue, and X. Zhou, "A new approach for measuring the resilience of transport infrastructure networks," Complexity, vol. 2020, Article ID 7952309, 16 pages, 2020.

[14] S. Enjalbert, V. Frédéric, M. Pichon, and K. A. Ouedraogo, "Assessment of transportation system resilience," in Human 
Modelling in Assisted Transportation, pp. 335-341, Springer, Heidelberg, Germany, 2011.

[15] D. Henry and J. Emmanuel Ramirez-Marquez, "Generic metrics and quantitative approaches for system resilience as a function of time," Reliability Engineering \& System Safety, vol. 99, pp. 114-122, 2012.

[16] A. Osei-Asamoah and N. Lownes, "Complex network method of evaluating resilience in surface transportation networks," Transportation Research Record: Journal of the Transportation Research Board, vol. 2467, no. 1, pp. 120-128, 2014.

[17] M. Ouyang, "Review on modeling and simulation of interdependent critical infrastructure systems," Reliability Engineering \& System Safety, vol. 121, pp. 43-60, 2014.

[18] R. Faturechi and E. Miller-Hooks, "Travel time resilience of roadway networks under disaster," Transportation Research Part B: Methodological, vol. 70, pp. 47-64, 2014.

[19] M. Janić, "Reprint of "Modelling the resilience, friability and costs of an air transport network affected by a large-scale disruptive event," Transportation Research Part A: Policy and Practice, vol. 81, pp. 77-92, 2015.

[20] D. R. Wuellner, S. Roy, and R. M. D’Souza, "Resilience and rewiring of the passenger airline networks in the United States," Physical Review E, vol. 82, no. 5, Article ID 056101, 2010.

[21] A. Cardillo, M. Zanin, J. Gómez-Gardeñes, M. Romance, A. J. García del Amo, and S. Boccaletti, "Modeling the multilayer nature of the European Air Transport Network: resilience and passengers re-scheduling under random failures," The European Physical Journal Special Topics, vol. 215, no. 1, pp. 23-33, 2013.

[22] O. Woolley-Meza, C. Thiemann, D. Grady et al., "Complexity in human transportation networks: a comparative analysis of worldwide air transportation and global cargo-ship movements," The European Physical Journal B, vol. 84, no. 4, pp. 589-600, 2011.

[23] R. Pant, K. Barker, J. E. Ramirez-Marquez, and C. M. Rocco, "Stochastic measures of resilience and their application to container terminals," Computers \& Industrial Engineering, vol. 70, pp. 183-194, 2014.

[24] H. Baroud, K. Barker, J. E. Ramirez-Marquez, and C. M. Rocco S., "Importance measures for inland waterway network resilience," Transportation Research Part E: Logistics and Transportation Review, vol. 62, pp. 55-67, 2014.

[25] R. Faturechi, E. Levenberg, and E. Miller-Hooks, "Evaluating and optimizing resilience of airport pavement networks," Computers \& Operations Research, vol. 43, pp. 335-348, 2014.

[26] F. Rouhana and D. Jawad, "Transportation network resilience against failures: GIS-based assessment of network topology role," International Journal of Disaster Resilience in the Built Environment, 2020.

[27] Z. Wang, A. P. C. Chan, J. Yuan, and B. Xia, "Recent advances in modeling the vulnerability of transportation networks," Journal of Infrastructure Systems, vol. 21, no. 2, Article ID 06014002, 2015.

[28] P. M. Orencio and M. Fujii, "A localized disaster-resilience index to assess coastal communities based on an analytic hierarchy process (AHP)," International Journal of Disaster Risk Reduction, vol. 3, pp. 62-75, 2013.

[29] L. Zhang, G. Zeng, D. Li, H.-J. Huang, H. E. Stanley, and S. Havlin, "Scale-free resilience of real traffic jams," Proceedings of the National Academy of Sciences, vol. 116, no. 18, pp. 8673-8678, 2019.
[30] W. H. Ip and D. Wang, "Resilience and friability of transportation networks: evaluation, analysis and optimization," IEEE Systems Journal, vol. 5, no. 2, pp. 189-198, 2011.

[31] U. Bhatia, D. Kumar, E. Kodra, and A. R. Ganguly, "Network science based quantification of resilience demonstrated on the Indian Railways Network," PLoS One, vol. 10, no. 11, Article ID e0141890, 2015.

[32] E. Miller-Hooks, X. Zhang, and R. Faturechi, "Measuring and maximizing resilience of freight transportation networks," Computers \& Operations Research, vol. 39, no. 7, pp. 16331643, 2012.

[33] X. Xue, L. Wang, and R. J. Yang, "Exploring the science of resilience: critical review and bibliometric analysis," Natural Hazards, pp. 1-34, 2018.

[34] D. Paton, M. Millar, and D. Johnston, "Community resilience to volcanic hazard consequences," Natural Hazards, vol. 24, no. 2, pp. 157-169, 2001.

[35] L. Wang, X. Xue, Y. Zhang, and X. Luo, "Exploring the emerging evolution trends of urban resilience research by scientometric analysis," International Journal of Environmental Research and Public Health, vol. 15, no. 10, p. 2181, 2018.

[36] C. Folke, S. Carpenter, T. Elmqvist, L. Gunderson, C. S. Holling, and B. Walker, "Resilience and sustainable development: building adaptive capacity in a world of transformations," AMBIO: A Journal of the Human Environment, vol. 31, no. 5, pp. 437-440, 2002.

[37] C. S. Holling, "Resilience and stability of ecological systems," Annual Review of Ecology and Systematics, vol. 4, no. 1, pp. 1-23, 1973.

[38] T. McDaniels, S. Chang, D. Cole, J. Mikawoz, and H. Longstaff, "Fostering resilience to extreme events within infrastructure systems: characterizing decision contexts for mitigation and adaptation," Global Environmental Change, vol. 18, no. 2, pp. 310-318, 2008.

[39] X. Ma, H. Yu, Y. Wang, and Y. Wang, "Large-scale transportation network congestion evolution prediction using deep learning theory," PLoS One, vol. 10, no. 3, Article ID e0119044, 2015.

[40] S. Zheng and R. Du, "How does urban agglomeration integration promote entrepreneurship in China? Evidence from regional human capital spillovers and market integration," Cities, vol. 97, Article ID 102529, 2020.

[41] X. Dong, S. Zheng, and M. E. Kahn, "The role of transportation speed in facilitating high skilled teamwork across cities," Journal of Urban Economics, vol. 115, Article ID 103212, 2020.

[42] M. E. O'Kelly, "Network hub structure and resilience," Networks and Spatial Economics, vol. 15, no. 2, pp. 235-251, 2015.

[43] X. Xu, A. Chen, S. Jansuwan, C. Yang, and S. Ryu, “Transportation network redundancy: complementary measures and computational methods," Transportation Research Part B: Methodological, vol. 114, pp. 68-85, 2018.

[44] S. Hosseini, K. Barker, and J. E. Ramirez-Marquez, "A review of definitions and measures of system resilience," Reliability Engineering \& System Safety, vol. 145, pp. 47-61, 2016.

[45] A. Reggiani, "Network resilience for transport security: some methodological considerations," Transport Policy, vol. 28, pp. 63-68, 2013.

[46] X. Zhang and E. Miller-Hooks, "Scheduling short-term recovery activities to maximize transportation network resilience," Journal of Computing in Civil Engineering, vol. 29, no. 6, Article ID 04014087, 2014. 
[47] Y. Liu, S. McNeil, J. Hackl, and B. T. AdeyB. T., "Prioritizing transportation network recovery using a resilience measure," Sustainable and Resilient Infrastructure, pp. 1-12, 2020.

[48] R. Guidotti, H. Chmielewski, V. Unnikrishnan, P. Gardoni, T. McAllister, and J. van de Lindt, "Modeling the resilience of critical infrastructure: the role of network dependencies," Sustainable and Resilient Infrastructure, vol. 1, no. 3-4, pp. 153-168, 2016.

[49] S. D. Solares, "Probing viscoelastic surfaces with bimodal tapping-mode atomic force microscopy: underlying physics and observables for a standard linear solid model," Beilstein Journal of Nanotechnology, vol. 5, no. 1, pp. 1649-1663, 2014.

[50] L. Wang, X. Xue, W. Xue, and H. Yuan, "Measuring the resilience of China railway network," in Proceedings of the Construction Research Congress 2020: Infrastructure Systems and Sustainability, American Society of Civil Engineers, Reston, VA, USA, November 2020. 